\title{
The Role of the Serum 25-OH Vitamin D Level in Detecting Prostate Cancer in Men with Elevated Prostate-Specific Antigen Levels
}

\section{Min Hyuk Kim}

Seoul National University

\section{Sangjun Yoo}

Seoul National University Boramae Medical Center

\section{Min Soo Choo}

Seoul National University Boramae Medical Center

Min Chul Cho

Seoul National University Boramae Medical Center

\section{Hwancheol Son}

Seoul National University Boramae Medical Center

Hyeon Jeong ( $\sim$ drjeongh@gmail.com )

Seoul National University Boramae Medical Center

\section{Research Article}

Keywords: vitamin D, prostate biopsy, histopathological results, cancer diagnosis.

Posted Date: January 6th, 2022

DOI: https://doi.org/10.21203/rs.3.rs-1206468/v1

License: (9) (i) This work is licensed under a Creative Commons Attribution 4.0 International License. Read Full License 


\section{Abstract}

We aimed to determine whether vitamin $\mathrm{D}$ levels before prostate biopsy have diagnostic value for clinically significant prostate cancer. The study cohort included patients who underwent prostate biopsy. A total of 224 patients were enrolled in our study, and serum vitamin D levels were measured from February 2016 to December 2019 in routine laboratory tests. To determine the relationship between vitamin D levels and the aggressiveness of prostate cancer, we used multivariate analysis. Based on the histopathological results, the serum vitamin D level was marginally lower in the group with higher positive cores and pT3 or higher, and the serum vitamin D level was significantly lower in the large tumor volume group. In the univariate analysis, the prostate cancer diagnosis rate was associated with low vitamin $D$ levels. In clinically significant prostate cancer diagnosis, low vitamin D levels were found in the univariate (odds ratio [OR], 0.955; $\mathrm{P}<0.001)$ and multivariate ( $\mathrm{OR}, 0.944 ; \mathrm{P}=0.027)$ analyses. In conclusion, we found that the incidence of prostate cancer tends to increase as the vitamin D level is lower in the Asian population, and this is particularly helpful in diagnosing clinically significant prostate cancer.

\section{Introduction}

Prostate cancer is the second most common cancer diagnosed in men worldwide, with more than 1 million cases occurring each year ${ }^{1}$. In the United States, prostate cancer is the most common cancer in men, accounting for $26 \%$ of cancer diagnoses ${ }^{2}$. In addition, the incidence and prevalence of prostate cancer have recently increased in Asian countries ${ }^{3}$. In Korea, the incidence and prevalence of prostate cancer became fourth and third, respectively, in 2018. With the discovery of prostate-specific antigen (PSA) in the 1970s, the diagnosis rate has increased through screening for prostate cancer. However, because of the widespread use of PSA, there has been no significant improvement in screening studies for detecting prostate cancer, and a novel diagnostic test for prostate cancer detection is awaited.

In addition, because the current clinical guidelines suggest active surveillance as a preferred treatment option for low-risk prostate cancer, accurately detecting clinically significant prostate cancer, which needs to be treated at an appropriate time, has become more important than ever before. Although multiparametric magnetic resonance imaging (MRI) and MRI fusion biopsy have recently gained attention for detecting clinically significant prostate cancer, due to the high cost and accessibility issues, there is a need for more cost-effective and easy methods to reduce unnecessary prostate biopsy. To this end, several studies have been conducted for appropriate predictive value, but diagnostic parameters with high sensitivity and specificity have not yet been determined ${ }^{4}$.

Recent studies have shown that vitamin D deficiency is linked to several types of cancer, including breast, colon, rectum, stomach, and esophageal cancers ${ }^{5-8}$. Several studies have shown that vitamin $D$ levels and cancer incidence are negatively correlated and that vitamin $D$ is related to overall survival and disease-free survival. These anticancer effects of vitamin $D$ are mediated by the regulation of specific signaling pathways via vitamin D receptors (VDRs). The activated form of vitamin D, calcitriol, binds to VDR to promote cell differentiation and apoptosis and inhibit cellular proliferation, angiogenesis, and 
tumor cell invasion ${ }^{9-12}$. In addition, vitamin $\mathrm{D}$ also affects the immune system, causing antiinflammatory reactions and suppressing cancer progression ${ }^{13-15}$.

Several studies have revealed a negative relationship between vitamin D and prostate cancer. Moreover, a previous study revealed a negative relationship between vitamin D and Gleason score (GS), which would be useful for prostate cancer aggressiveness evaluation ${ }^{16-19}$. Although the association between prostate cancer and vitamin D has been reported, few studies have investigated the diagnostic value of vitamin $D$ levels in clinically significant prostate cancer. Therefore, in the current study, we aimed to reveal the role of serum vitamin $D$, the precursor for calcitriol, in the detection of prostate cancer, especially in the detection of clinically significant prostate cancer. In addition, we assessed the relationship between vitamin D levels and the results of multiparametric MRI to assess the additive value of vitamin $D$ on MRI in men with elevated PSA levels.

\section{Materials And Methods}

We established a study cohort of patients who underwent transrectal ultrasound (TRUS)-guided biopsy after outpatient treatment at the Department of Urology at Boramae Medical Center, Seoul, Republic of Korea. These patients consistently had a PSA level of $\geq 3 \mathrm{ng} / \mathrm{mL}$, had suspected cancer lesions on imaging tests, or had nodules on digital rectal examination. From December 2015 to December 2019, 1,500 patients underwent TRUS biopsy, among whom patients previously treated for prostate cancer and patients suspected of regional lymph node metastasis or distant metastasis on the imaging test were excluded. Finally, 224 individuals who allowed their serum vitamin D levels to be measured before prostate biopsy were included in our study. This study was approved by the Seoul Metropolitan Government-Seoul National University (SMG-SNU) Boramae Medical Center Institutional Review Board (IRB number: 10-2021-123). All experimental methods were carried out in accordance with relevant guidelines and regulations (the ethical standards of the 1964 Declaration of Helsinki and its later amendments). Since current study is only based on clinical records of patients collected by a retrospective method, informed consent was waived by SMG-SNU Boramae Medical Center Institutional Review Board.

Prostate cancer with a GS of $\geq 7$ was defined as clinically significant prostate cancer. In these patients, standard 12-core TRUS-guided prostate biopsy was routinely performed, although for areas where prostate cancer was strongly suspected on imaging studies, one to two biopsies were additionally performed. In these patients, serum vitamin $D$ levels were routinely measured $1-4$ weeks before prostate biopsy. Because there was seasonal variation in the serum vitamin D levels, patients were first divided according to the season. Of the 224 patients, 101 underwent prostate biopsy in the spring, 40 in the summer, 24 in the fall, and 59 in the winter. However, there was no seasonal difference in the patients in our study $(\mathrm{P}=0.551)$, which may be due to increased indoor activities and increased oral intake of vitamin D (Supplement 1). In this regard, we decided to conduct the analysis regardless of the season. For the Cox regression analysis, the vitamin $\mathrm{D}$ level was used as a continuous variable. To compare the serum vitamin $\mathrm{D}$ levels according to pathohistological results, the ratio of the total number of cores to the 
number of positive cores was calculated and divided into high and low groups with an average value of $43.4 \%$ of TRUS biopsy. The serum vitamin $\mathrm{D}$ levels were compared by dividing the final pathology result, tumor volume, lymphatic invasion, and perineural invasion factor in 36 patients who underwent radical prostatectomy (RP). The final pathology result was based on $\mathrm{pT} 3$, and the tumor volume ratio was divided into large and small groups based on an average value of $17.2 \%$.

Prostate MRI was performed with Ingenia CX 3.0T MP MRI for high resolution. MRI was performed in all patients with prostate cancer after the histological diagnosis of prostate cancer, and suspicious lesions on MRI were checked by a urology-specialized radiologist if there was a lesion with a PI-RADS version 2 score of $\geq 3$.

The distribution of patient characteristics was compared using Student's t test, Pearson's $\chi^{2}$ test, or Fisher's exact test. We present the demographic and clinical characteristics of patients by detecting prostate cancer. Continuous factors are expressed as the means and standard deviations, and categorical factors are expressed as percentages. In addition, the detection rate of prostate cancer and clinically significant prostate cancer according to vitamin D levels was presented and compared. We performed logistic regression models for univariate and multivariate analyses to determine the relationship between prostate cancer and clinically significant prostate cancer and vitamin D levels, in addition to other variables, including age, body mass index (BMI), hypertension (HTN), diabetes, serum PSA level, and prostate volume. All statistical comparisons were performed using IBM SPSS Statistics version 21. Statistical significance was set at $\mathrm{P}<0.05$.

\section{Results}

Age (BPH 66.5 vs. insignificant 69.3 vs. significant 69.2, $\mathrm{P}=0.049$ ) and PSA (BPH $7.9 \mathrm{ng} / \mathrm{mL}$ vs. insignificant $9.7 \mathrm{ng} / \mathrm{mL}$ vs. significant $34.2 \mathrm{ng} / \mathrm{mL}, P<0.001$ ) were higher in the prostate cancer group, and the prostate total volume (BPH $48.9 \mathrm{~mL}$ vs. insignificant $34.2 \mathrm{~mL}$ vs. significant $35.0 \mathrm{~mL}, \mathrm{P}<0.001$ ) was lower in men with prostate cancer than in men without prostate cancer. In particular, the clinically significant prostate cancer group had significantly higher PSA levels than the BPH group and clinically insignificant prostate cancer group. The HTN factor (BPH $40.1 \%$ vs. insignificant $55.0 \%$ vs. significant $55.7 \%, P=0.085$ ) and diabetes factors (BPH $13.4 \%$ vs. insignificant $30.0 \%$ vs. significant $24.6 \%, P=0.055$ ) also showed marginal differences between the three groups. The serum vitamin $D$ levels were lower in the prostate cancer group, but the difference was not statistically significant (BPH $19.6 \mathrm{ng} / \mathrm{mL}$ vs. insignificant $19.1 \mathrm{ng} / \mathrm{mL}$ vs. significant $18.1 \mathrm{ng} / \mathrm{mL}, \mathrm{P}=0.562$ ) (Table 1 ).

In TRUS biopsy, the ratio of the total number of cores to the number of positive cores was calculated. The serum vitamin $D$ levels were marginally lower in the group with a high positive core ratio than in the group with a low positive core ratio (high group $16.3 \mathrm{ng} / \mathrm{mL}$ vs. low group $19.5 \mathrm{ng} / \mathrm{mL}, \mathrm{P}=0.087$ ). Of the 82 patients with prostate cancer, 36 underwent RP, and the remaining patients underwent radiotherapy or androgen deprivation therapy. According to the histopathological results after surgery, the serum vitamin D level was marginally lower in patients with pT3 or higher than in patients with $\mathrm{pT} 2$ or lower (T2 $\geq 19.1$ 
$\mathrm{ng} / \mathrm{mL}$ vs. T3 $\leq 15.0 \mathrm{ng} / \mathrm{mL}, \mathrm{P}=0.089)$. The serum vitamin $\mathrm{D}$ level was low in the group with a large volume ratio of prostate cancer (large group $11.2 \mathrm{ng} / \mathrm{mL}$ vs. small group $19.2 \mathrm{ng} / \mathrm{mL}, P<0.001$ ). In the presence of lymphatic and perineural invasions, the serum vitamin $D$ levels were lower in the invasion group, but the difference was not statistically significant (lymphatic invasion (-) $18.2 \mathrm{ng} / \mathrm{mL}$ vs. lymphatic invasion (+) $14.8 \mathrm{ng} / \mathrm{mL}, \mathrm{P}=0.278$; perineural invasion $(-) 19.3 \mathrm{ng} / \mathrm{mL}$ vs. perineural invasion (+) $16.7 \mathrm{ng} / \mathrm{mL}, \mathrm{P}=0.300$ ) (Table 2).

In the univariate analysis, the prostate cancer diagnosis rate was significantly increased with low vitamin $D$ levels (odds ratio [OR], 0.975; $\mathrm{P}<0.001$ ) and other factors, such as small prostate total volume (OR, 0.983; $P<0.001)$, young age (OR, 0.993; $P<0.001)$, low $B M I(O R, 0.978 ; P<0.001)$, and high serum $P S A$ levels $(\mathrm{OR}, 1.063 ; \mathrm{P}<0.001)$. In the multivariate analysis, the prostate cancer diagnosis rate was significantly increased with high serum PSA levels $(O R, 1.083 ; P=0.003)$ and small prostate total volume $(O R, 0.937 ; P<0.001)$. The serum vitamin $D$ levels were not statistically significant but were marginally associated with the prostate cancer diagnosis rate $(\mathrm{OR}, 0.963 ; \mathrm{P}=0.094)$ (Table 3$)$.

We also analysed the correlation between the vitamin D level and clinically significant prostate cancer diagnosis. In the univariate analysis, the clinically significant prostate cancer diagnosis rate was significantly increased with low vitamin $D$ levels $(O R, 0.955 ; P<0.001)$ and other factors, such as small prostate total volume (OR, 0.974; $P<0.001)$, young age (OR, 0.987; $P<0.001)$, low $B M I(O R, 0.962 ; P<0.001)$, and no HTN (OR, 0.500; $P=0.001)$. In the multivariate analysis, the clinically significant prostate cancer diagnosis rate was significantly increased with low vitamin $D$ levels $(O R, 0.944 ; P=0.027)$ and other factors, such as old age (OR, 1.026; $P=0.032)$, high PSA levels $(O R, 1.077 ; P=0.001)$, and small prostate total volume $(\mathrm{OR}, 0.933 ; \mathrm{P}<0.001)$ (Table 3$)$.

Since the correlation between prostate cancer and PSA is widely known, univariate and multivariate analyses were performed on patients with a serum PSA level of $\leq 20 \mathrm{ng} / \mathrm{mL}$. A total of 150 patients were included in this study. In all prostate cancers, the diagnosis rate was significantly increased with low vitamin $D$ levels $(O R, 0.963 ; P<0.001)$ as a result of the univariate analysis. In the multivariate analysis, the diagnosis rate was marginally increased with low vitamin $D$ levels $(O R, 0.957 ; P=0.083)$ and other factors, such as small prostate size $(O R, 0.917 ; P<0.001)$ and old age $(O R, 2.469 ; P=0.039)$. For clinically significant prostate cancer, the univariate analysis showed that the diagnosis rate was significantly increased with low vitamin $D$ levels $(O R, 0.942 ; P<0.001)$. In the multivariate analysis, the diagnosis rate was marginally increased with low vitamin $D$ levels $(O R, 0.942 ; P=0.053)$ and other factors, such as small prostate size $(\mathrm{OR}, 0.908 ; \mathrm{P}<0.001)$.

Table 1. Demographic and clinical characteristics of patients 


\begin{tabular}{|lllll|}
\hline & BPH $(\boldsymbol{n}=142)$ & Insignificant $(\boldsymbol{n = 2 0})$ & Significant $(\boldsymbol{n}=\mathbf{6 2})$ & $\boldsymbol{p}$ value \\
\hline Age $(\mathrm{years})$ & $66.5 \pm 7.9$ & $69.3 \pm 7.2$ & $69.2 \pm 8.7$ & 0.049 \\
\hline BMI $(\mathrm{kg} / \mathrm{m} 2)$ & $24.8 \pm 2.9$ & $23.9 \pm 2.3$ & $24.4 \pm 3.3$ & 0.383 \\
\hline Hypertension $(\mathrm{n}, \%)$ & $57(40.1)$ & $11(55.0)$ & $34(55.7)$ & 0.085 \\
\hline Diabetes $(\mathrm{n}, \%)$ & $19(13.4)$ & $6(30.0)$ & $15(24.6)$ & 0.055 \\
\hline WBC $\left(\times 10^{3} / \mu \mathrm{l}\right)$ & $6.1 \pm 2.6$ & $5.5 \pm 1.2$ & $6.1 \pm 1.5$ & 0.561 \\
\hline ESR $(\mathrm{mm} / \mathrm{h})$ & $11.9 \pm 11.2$ & $6.2 \pm 7.2$ & $14.9 \pm 15.2$ & 0.328 \\
\hline CRP $(\mathrm{mg} / \mathrm{L})$ & $0.36 \pm 1.08$ & $0.11 \pm 0.14$ & $0.28 \pm 0.65$ & 0.679 \\
\hline PSA $(\mathrm{ng} / \mathrm{mL})$ & $7.9 \pm 5.7$ & $9.7 \pm 8.3$ & $34.2 \pm 66.7$ & $<0.001$ \\
\hline Prostate total volume $(\mathrm{g})$ & $48.9 \pm 22.8$ & $34.2 \pm 18.8$ & $35.0 \pm 16.1$ & $<0.001$ \\
\hline 25-OH-VitD level $(\mathrm{ng} / \mathrm{mL})$ & $19.6 \pm 10.0$ & $19.1 \pm 7.5$ & $18.1 \pm 8.5$ & 0.562 \\
\hline
\end{tabular}

BMI: body mass index, WBC: white blood cell, ESR: erythrocyte sedimentation rate, CRP: c-reactive protein, PSA: prostate-specific antigen 
Table 2

Serum vitamin D level according to pathohistological results

\begin{tabular}{|lll|}
\hline & 25-OH-VitD level $(\mathrm{ng} / \mathrm{mL})$ & $p$ value \\
\hline Positive/Total core $(\%)$ & & 0.087 \\
\hline Low $(n=51)$ & $19.5 \pm 7.4$ & \\
\hline High $(n=31)$ & $16.3 \pm 9.2$ & 0.089 \\
\hline Pathology & & \\
\hline T2 $\geq(n=23)$ & $19.1 \pm 7.1$ & $<0.001$ \\
\hline T3 $\leq(n=13)$ & $15.0 \pm 6.0$ & \\
\hline Tumor volume $(\%)$ & & \\
\hline Small $(n=29)$ & $19.2 \pm 6.8$ & \\
\hline Large $(n=7)$ & $11.2 \pm 3.2$ & \\
\hline Lymphatic invasion & & \\
\hline No $(n=30)$ & $18.2 \pm 7.2$ & \\
\hline Yes $(n=6)$ & $14.8 \pm 5.2$ & \\
\hline Perineural invasion & & \\
\hline No $(n=13)$ & $19.3 \pm 8.1$ & \\
\hline Yes $(n=23)$ & $16.7 \pm 6.2$ & \\
\hline
\end{tabular}

Table 3. Univariate and multivariate logistic regression analysis of Asian patients with prostate biopsy ( $n$ $=171)$

(A) Logistic regression analysis according to Gleason score 6 


\begin{tabular}{|lllll|}
\hline & $\begin{array}{l}\text { Univariate (OR 95\% } \\
\text { Cl) }\end{array}$ & p value & $\begin{array}{l}\text { Multivariate (OR 95\% } \\
\text { Cl) }\end{array}$ & $p$ value \\
\hline $\begin{array}{l}\text { Age (continuous } \\
\text { variable) }\end{array}$ & $\begin{array}{l}0.993(0.989- \\
0.997)\end{array}$ & $<0.001$ & $1.064(1.012-1.118)$ & 0.015 \\
\hline $\begin{array}{l}\text { BMI (continuous } \\
\text { variable) }\end{array}$ & $\begin{array}{l}0.978(0.968- \\
0.989)\end{array}$ & $<0.001$ & & \\
HTN (yes vs no) & $\begin{array}{l}0.821(0.556- \\
1.213)\end{array}$ & 0.323 & & \\
DM (yes vs no) & $\begin{array}{l}1.105(0.594- \\
2.056)\end{array}$ & 0.752 & & \\
\hline PSA (continuous & $\begin{array}{l}1.063(1.027- \\
\text { variable) }\end{array}$ & $<0.100)$ & $1.083(1.028-1.140)$ & 0.003 \\
\hline Prostate total volume & $\begin{array}{l}0.983(0.976- \\
0.990)\end{array}$ & $<0.001$ & $0.937(0.910-0.965)$ & $<0.001$ \\
(continuous variable) & & & & \\
\hline 25-OH-VitD level & $0.975(0.962-$ & $<0.001$ & $0.963(0.922-1.006)$ & 0.094 \\
(continuous variable) & $0.988)$ & & & \\
\hline
\end{tabular}

(B) Logistic regression analysis according to Gleason score 7 (clinically significant prostate cancer) HTN: hypertension, DM: diabetes mellitus

\begin{tabular}{|lllll|}
\hline & Univariate (OR 95\% Cl) & $p$ value & Multivariate (OR 95\% Cl) & $p$ value \\
\hline Age (continuous variable) & $0.987(0.982-0.991)$ & $<0.001$ & $1.026(1.002-1.050)$ & 0.032 \\
\hline BMI (continuous variable) & $0.962(0.950-0.973)$ & $<0.001$ & & \\
\hline HTN (yes vs no) & $0.500(0.331-0.755)$ & 0.001 & & \\
\hline DM (yes vs no) & $0.600(0.316-1.138)$ & 0.118 & & 0.001 \\
\hline PSA (continuous variable) & $1.064(1.031-1.099)$ & $<0.001$ & $1.077(1.031-1.125)$ & $<0.001$ \\
\hline Prostate total volume & $0.974(0.965-0.982)$ & $<0.001$ & $0.933(0.902-0.966)$ & \\
(continuous variable) & & & & 0.027 \\
\hline 25-OH-VitD level & $0.955(0.941-0.969)$ & $<0.001$ & $0.944(0.897-0.993)$ & \\
(continuous variable) & & & & \\
\hline
\end{tabular}

\section{Discussion}

Currently, PSA is used as a screening test to diagnose prostate cancer, and TRUS biopsy is performed as a confirmation test. In a previous meta-analysis study, the sensitivity $(72.1 \%)$ and positive predictive value 
(25.1\%) of PSA for prostate cancer diagnosis were not high ${ }^{20}$. According to the results of recently conducted large randomized clinical trials, the proportion of clinically insignificant prostate cancer diagnosed by PSA screening was high, and as a result, several unnecessary TRUS biopsies were performed ${ }^{21}$. To overcome this, prostate MRI is performed before prostate biopsy, and MRI fusion biopsy is performed if there is a target lesion, albeit with high cost and accessibility issues.

Prostate cancer is a slow-growing cancer, and asymptomatic prostate cancer is found in several individuals at autopsy. For patients with clinically insignificant prostate cancer, active surveillance is implemented to minimize the side effects of treatment without affecting their survival. With an increase in the number of patients undergoing active surveillance, it is becoming increasingly important to accurately diagnose patients with clinically significant prostate cancer who require treatment at an appropriate time. In the current study, we revealed that serum vitamin D may be a helpful indicator for improving the detection rate of clinically significant prostate cancer. In other words, although further larger studies are needed for confirmation, serum vitamin D may be a useful way to reduce unnecessary TRUS biopsy and increase the diagnosis rate of clinically significant prostate cancer.

Based on our study findings, there was a significant negative correlation between vitamin $D$ and clinically significant prostate cancer ( $O R, 0.944 ; P=0.027)$. Likewise, other studies have shown a negative relationship between vitamin D and prostate cancer malignancy. In a nested case-control study, Schenk et al. ${ }^{18}$ recruited and analysed 1,700 cases and controls. The serum vitamin $D$ levels were divided into quartiles and compared, and multiple regression analysis was performed by adjusting for age and race. It was found that the higher the vitamin $D$ level was, the lower the risk of a GS of 8-10 (quartile 4 vs. 1; OR, $0.55 ; \mathrm{P}=0.04$ ). In a prospective study, Ahn et al. ${ }^{22}$ compared 749 patients with prostate cancer with 781 control individuals and concluded that a lower vitamin $D$ level did not increase the prostate cancer risk but could increase the aggressiveness of prostate cancer (GS sum of $\geq 7$ or clinical stage III or IV). Nyame et al. ${ }^{19}$ analysed the correlation between the serum vitamin $D$ level and prostate cancer aggressiveness using multiple regression analysis in 190 patients who underwent RP. Adverse pathology was defined as dominant Gleason pattern 4, the presence of any pattern 5, and extracapsular extension based on histopathology after surgery. Multiple regression analysis showed that the risk of adverse pathology was increased in the group with a serum vitamin $D$ level of $\leq 30 \mathrm{ng} / \mathrm{mL}(\mathrm{OR}, 2.64 ; \mathrm{P}=0.01)$.

In addition to in vitro studies, in vivo studies have shown the relationship between vitamin $D$ and prostate cancer in human or animal cells. Oades et al. ${ }^{23}$ showed that VDR expression and vitamin D inhibited cancer cell growth in rodent cells, and Peehl et al. ${ }^{24}$ showed antiproliferative and differentiating action of vitamin $D$ in human prostate cancer cells. The anticancer effect of vitamin $D$ is unclear, but animal studies have shown that it inhibits angiogenesis ${ }^{25,26}$. Based on these results, vitamin $D$ can be assumed to inhibit tumor cell proliferation by inhibiting angiogenesis in prostate cancer cells. In addition, there is a theory that vitamin D binds to VDR and directly induces cell cycle arrest, thereby inhibiting tumor cell proliferation. In our study, the serum vitamin $D$ level was higher in the group with a small tumor volume, which can be seen as a result of angiogenesis inhibition or cell cycle arrest through VDR (Table 2). The 
results of in vitro and in vivo studies support the results of our study, and vitamin D may be used as a tool to prevent clinically significant prostate cancer by reducing prostate malignancies. Moreover, vitamin $\mathrm{D}$ may be a helpful marker for detecting clinically significant prostate cancer.

The correlation between PSA and prostate cancer is well known, and excessively high PSA levels may reduce the effect of serum vitamin $D$ factor. We set the standard PSA level to $\leq 20 \mathrm{ng} / \mathrm{mL}$ and performed univariate and multivariate analyses. The analysis revealed that the serum vitamin $\mathrm{D}$ level had a marginal effect on the diagnosis of all prostate cancers and clinically significant cancers. Few patients were included in the analysis due to PSA restrictions, and a large-scale analysis is required in the future.

To increase the diagnosis rate of clinically significant prostate cancer that requires treatment, prostate MRI is performed before TRUS biopsy. For patients with high PSA levels, it is useful to obtain an accurate target lesion through prostate MRI and perform MRI fusion biopsy. However, for patients with slightly elevated PSA levels, MRI scans that are not covered by health insurance can be a financial burden to the patient, and there are cases where it is difficult to distinguish between prostate cancer and normal prostate tissue even though standardized interpretation systems, such as PI-RADS version 2, have been adapted. Based on this study, the higher the vitamin D level was, the more target lesions were observed on prostate MRI (Supplement 2). In other words, radiology specialists could observe the target lesion more clearly by distinguishing the boundary between prostate cancer and normal prostate by the antiinflammatory effect of vitamin D. Although the relationship between serum vitamin D levels and MRI results needs to be validated in future studies, MRI may be recommended for patients with slightly elevated PSA levels and elevated serum vitamin D levels.

There are certain limitations to the current study, including the small number of patients. In addition, this study is a nested case-control study, and the temporal causality is unclear, with a possibility of selection and recall bias. The effect of vitamin $D$ level on the incidence of prostate cancer can be studied using prospective cohort studies in the future. Since the vitamin $D$ level in the body changes with exposure to ultraviolet radiation, seasonal variation should be considered. In previous studies, there was a significant seasonal difference in vitamin D levels. However, in our study, there was no significant difference in the vitamin D level according to the season (Supplement 1). In modern society, with the development of the culture of living indoors and an increase in the number of individuals taking multivitamins, vitamin $\mathrm{D}$ levels do not vary greatly according to ultraviolet radiation.

\section{Conclusion}

To date, most studies on prostate cancer and vitamin D have been conducted in European Americans and African Americans with a small number of patients. In this study, we found that the incidence of prostate cancer tends to increase with lower vitamin D levels in the Asian population, and it is particularly helpful in diagnosing clinically significant prostate cancer.

\section{Declarations}




\section{Author contributions}

MH Kim: Data collection or management, Data analysis, Manuscript writing/editing

S Yoo: Protocol/project development, Data collection or management, Manuscript editing

MS Choo: Data collection, Manuscript editing

MC Cho: Data collection, Data analysis

H Son: Data collection

H Jeong: Protocol/project development, Data collection

\section{Funding}

There was no funding received for conducting this study.

\section{Conflict of interest}

The authors have no conflicts of interest to declare that they are relevant to the content of this article.

\section{Ethical approval}

The study was approved by the Ethics Committee of the Seoul National University Boramae Medical Center (10-2021-123).

\section{Consent to participate and for publication}

Data were collected retrospectively from clinical files and anonymized for analysis. No specific consent was obtained from all patients.

\section{References}

1 Bray, F. et al. Global cancer statistics 2018: GLOBOCAN estimates of incidence and mortality worldwide for 36 cancers in 185 countries. CA: a cancer journal for clinicians68, 394-424 (2018).

2 Siegel, R. L., Miller, K. D., Fuchs, H. E. \& Jemal, A. Cancer Statistics, 2021. CA Cancer J Clin71, 7-33, doi:10.3322/caac.21654 (2021).

3 Kimura, T. \& Egawa, S. Epidemiology of prostate cancer in Asian countries. International journal of urology25, 524-531 (2018).

4 Djavan, B. et al. PSA, PSA density, PSA density of transition zone, free/total PSA ratio, and PSA velocity for early detection of prostate cancer in men with serum PSA 2.5 to $4.0 \mathrm{ng} / \mathrm{mL}$. Urology $54,517-522$ (1999). 
5 Grant, W. B. Lower vitamin-D production from solar ultraviolet-B irradiance may explain some differences in cancer survival rates. Journal of the National Medical Association98, 357 (2006).

6 Garland, C. F. et al. Vitamin D and prevention of breast cancer: pooled analysis. J Steroid Biochem Mol Bio/103, 708-711, doi:10.1016/j.jsbmb.2006.12.007 (2007).

7 Wang, L., Wang, C., Wang, J., Huang, X. \& Cheng, Y. Longitudinal, observational study on associations between postoperative nutritional vitamin $\mathrm{D}$ supplementation and clinical outcomes in esophageal cancer patients undergoing esophagectomy. Sci Rep6, 38962, doi:10.1038/srep38962 (2016).

8 Garland, C. et al. Serum 25-hydroxyvitamin D and colon cancer: eight-year prospective study. The Lancet334, 1176-1178 (1989).

9 Hendrickson, W. K. et al. Vitamin D receptor protein expression in tumor tissue and prostate cancer progression. J Clin Onco/29, 2378-2385, doi:10.1200/JC0.2010.30.9880 (2011).

10 Xu, H.-M., Tepper, C. G., Jones, J. B., Fernandez, C. E. \& Studzinski, G. P. 1, 25-Dihydroxyvitamin D3 protects HL60 cells against apoptosis but down-regulates the expression of the bcl-2 gene. Experimental cell research209, 367-374 (1993).

11 Ingles, S. A. et al. Association of prostate cancer risk with genetic polymorphisms in vitamin D receptor and androgen receptor. Journal of the National Cancer Institute89, 166-170 (1997).

12 Taylor, J. A. et al. Association of prostate cancer with vitamin D receptor gene polymorphism. Cancer Research56, 4108-4110 (1996).

13 Krishnan, A. V. \& Feldman, D. Mechanisms of the anti-cancer and anti-inflammatory actions of vitamin D. Annual review of pharmacology and toxicology51, 311-336 (2011).

14 Patel, S. et al. Association between serum vitamin D metabolite levels and disease activity in patients with early inflammatory polyarthritis. Arthritis Rheum56, 2143-2149, doi:10.1002/art.22722 (2007).

15 Mellenthin, L. et al. Association between serum vitamin D concentrations and inflammatory markers in the general adult population. Metabolism63, 1056-1062, doi:10.1016/j.metabol.2014.05.002 (2014).

16 Ahonen, M. H., Tenkanen, L., Teppo, L., Hakama, M. \& Tuohimaa, P. Prostate cancer risk and prediagnostic serum 25-hydroxyvitamin D levels (Finland). Cancer Causes \& Contro/11, 847-852 (2000).

17 Murphy, A. B. et al. Vitamin D deficiency predicts prostate biopsy outcomes. Clinical Cancer Research20, 2289-2299 (2014).

18 Schenk, J. M. et al. Serum 25-hydroxyvitamin D concentrations and risk of prostate cancer: results from the Prostate Cancer Prevention Trial. Cancer Epidemiol Biomarkers Prev23, 1484-1493, doi:10.1158/1055-9965.EPI-13-1340 (2014). 
19 Nyame, Y. A. et al. Associations Between Serum Vitamin D and Adverse Pathology in Men Undergoing Radical Prostatectomy. J Clin Onco/34, 1345-1349, doi:10.1200/JC0.2015.65.1463 (2016).

20 Mistry, K. \& Cable, G. Meta-analysis of prostate-specific antigen and digital rectal examination as screening tests for prostate carcinoma. The Journal of the American Board of Family Practice16, 95-101 (2003).

21 Crawford, E. D. et al. Comorbidity and mortality results from a randomized prostate cancer screening trial. Journal of Clinical Oncology29, 355 (2011).

22 Ahn, J. et al. Serum vitamin D concentration and prostate cancer risk: a nested case-control study. Journal of the National Cancer Institute100, 796-804 (2008).

23 Oades, G., Dredge, K., Kirby, R. \& Colston, K. Vitamin D receptor-dependent antitumour effects of 1, 25dihydroxyvitamin D3 and two synthetic analogues in three in vivo models of prostate cancer. $B J U$ internationa/90, 607-616 (2002).

24 Peehl, D. M. et al. Antiproliferative effects of 1, 25-dihydroxyvitamin D3 on primary cultures of human prostatic cells. Cancer Research54, 805-810 (1994).

$25 \mathrm{Maj}$, E. et al. Vitamin D derivatives potentiate the anticancer and anti-angiogenic activity of tyrosine kinase inhibitors in combination with cytostatic drugs in an A549 non-small cell lung cancer model. International journal of oncology52, 337-366 (2018).

26 Chung, I. et al. Role of vitamin D receptor in the antiproliferative effects of calcitriol in tumor-derived endothelial cells and tumor angiogenesis in vivo. Cancer research69, 967-975 (2009).

\section{Supplementary Files}

This is a list of supplementary files associated with this preprint. Click to download.

- Supplementary.docx 